\title{
Study on the Chemical Profiling of Fangfeng Tongsheng Pills by HPLC
}

\author{
J. LIU, S. Y. DAI, L. M. YE AND C. M. FU* \\ Department of Pharmaceutics Analysis, West China School of Pharmacy, Sichuan University, Chengdu-610 \\ 041, China
}

Liu, et al.: Chemical Profiling of Fangfeng Tongsheng Pills

\begin{abstract}
This study focused on profiling the major components of a complex traditional Chinese herbal preparation composed of seventeen herbs, Fangfeng Tongsheng pills by HPLC with photodiode array detection. Based on the optimized protocol, the HPLC fingerprint profiles were established. Within the chromatographic fingerprint, sixteen identified bioactive compounds belonging to iridoid, anthraquinones, flavonoids, triterpenoid, phenol, phenolic acids, alkaloid and chromones were successively separated and twelve was quantitatively determined. The linearity and range, limit of detection, and limit of quantitation, precision, accuracy and stability of the assay were validated according to Chinese Pharmacopoeia. The validated data showed that the method was suitable for quality evaluation of the valuable medicine listed in Chinese Pharmacopoeia by qualitative and quantitative information in one chromatographic fingerprint.
\end{abstract}

Key words: Fangfeng Tongsheng pills, HPLC, multicomponent quantification, Chinese herbal preparation

Traditional Chinese medicine (TCM) patent prescriptions have played an important role in the prevention and treatment of diseases for thousands of years in China. However, due to the complex chemical matrices of TCM patent prescriptions, the studies on pharmacology, toxicology and quality control are insufficient, so their safety and efficacy have not been widely recognized in most western countries. Therefore, it is necessary to put forward a reliable method to evaluate the quality of TCM patent prescriptions. A TCM patent prescription should be considered as a whole. However, the conventional approach was to determine only single or a few maker components, which only proved the existence of the ingredients, but could not indicate whether they were the real active ingredients of the preparation. Therefore, the results obtained are not convincing. Now, a method called 'multicomponent approach' has been put forward ${ }^{[1]}$ to represent the chemical profile of herbal medicines. This method is to simultaneously determine active components as many as possible. And it has been widely used to determine some major components in TCM patent prescriptions. In addition, fingerprint is also internationally accepted as an effective method to assess the overall quality of TCM patent prescriptions. It is used to emphasize the integrated chromatographic

*Address for correspondence E-mail: fuchunmei@scu.edu.cn profile and represent the systematic and comprehensive nature of TCM patent prescriptions ${ }^{[2]}$.

Fangfeng Tongsheng San (FTS) was first recorded in a book called Huang Di Suwen Xuanming lun ${ }^{[3]}$. It was composed of seventeen herbs (Radix Saposhnikoviae, Herba Schizonepetae, Mentha haplocalyx, Herba Ephedrae, liquorice, gardenia, Radix et Rhizoma Rhei, mirabilite, talc, Radix Platycodonis, gypsum, Rhizoma Ligustici Chuanxiong, Radix Angelicae Sinensis, white peony alba, Radix Scutellariae, Fructus Forsythiae and Rhizoma Atractylodis Macrocephalae (stir-fried). FTS was used to purge fire and remove toxicity. A lot of pharmacology researches on this prescription have been done by Japanese scientists since 1990s, and have confirmed that FTS had many new pharmacological effects, such as losing weight, reducing plasma lipids and fatty liver, treating arteriosclerosis and hyperlipidemia ${ }^{[4-7]}$. From the pharmacological researches, the efficacy is associated with its monarch drug, that is Herba Ephedrae,

This is an open access article distributed under terms of the Creative Commons Attribution-NonCommercial-ShareAlike 3.0 License, which allows other the remix, tweak, and build up to the non-commercially, as long as the author is credited and the new creations are licensed under the identical terms.

Accepted 05 Jun 2016

Revised 30 May 2016

Received 19 Aug 2015

Indian J Pharm Sci 2016;78(3):344-351 
Herba Schizonepetae, Radix Saposhnikoviae and Mentha haplocalyx, and its adjuvant drug, that is gardenia, mirabilite, talc, Radix Platycodonis, Radix Scutellariae, Fructus Forsythiae and Radix et Rhizoma Rhei. Fangfeng Tongsheng pills (FTP) is one of the new dosage forms to improve compliance of patients. Geniposide, ephedrine, 5-O-methylvisammioside and authentic reference herb, Radix et Rhizoma Rhei are applied in the quality evaluation of FTP using separate thin layer chromatography (TLC) techniques in Chinese Pharmacopeia 2010 edition (ChP.2010). It is tedious, unable to provide satisfactory coverage of other key chemical classes. More importantly, there is no quantitative analysis of this preparation. Some related work on FTP preparation by HPLC was reported, however, only single or a few components were determined in these papers ${ }^{[8-10]}$. Furthermore, the fundamental components of the monarch drugs were not included (i.e., ephedrine derived from Herba Ephedrae and 5-O-methylvisammioside derived from Radix Saposhnikoviae, respectively) which are the key active components. So these methods were inadequate and unable to evaluate the quality of the complex formulation.

Precondition of modernization quality control of TCM patent prescriptions is to make clear what the components are in prescription gradually. Only by this way can we further study the action mechanism of TCM patent prescriptions. 5-O-methylvisammioside, one of the chromones in Radix Saposhnikoviae, is authenticated to have an antipyretic effect and an anti-inflammatory activity ${ }^{[11]}$. Ephedrine, an alkaloid, has alpha and beta catecholamine effects ${ }^{[12]}$. Ligustrazine is the active component of Rhizoma Ligustici Chuanxiong, which has strong effect of block vasoconstriction and scavenges oxygen free radicals ${ }^{[13]}$. Geniposide, a kind of iridoid in Fructus Gardeniae, displays pharmacological action of anti-inflammatory and antithrombus ${ }^{[14]}$. Paeonol is a phenol which is used for treatment of many diseases such as eczema and pruritus ${ }^{[15]}$. Hesperidin, baicalin, and quercetin belong to flavonoids, which have the functions of antiinflammation, antiallergy, antivirus and anticancer and others ${ }^{[16]}$. The phenolic acids possess the antiinflammatory, anticarcinogenic, antibacterial and antiviral activities, like chlorogenic acid, glycyrrhizic acid and ferulic acid ${ }^{[17]} .5$ anthraquinones, aloe-emodin, emodin, chrysophanol, physcion and rhein, are main active components in rhubarb. Anthraquinones have purgative activities, analgesic effects, antibacterial effects, antitumor activity, prevention of hepatitis, antioxidant activity ${ }^{[18]}$. In this paper, 12 active components, ephedrine, geniposide, ferulic acid, 5-O-methylvisammioside, hesperidin, baicalin, paeonol, glycyrrhizic acid, aloe-emodin, rhein, chrysophanol and physcion, representing key chemical classes of the entire formulation were selected as markers for quantitative determination by HPLC. Another 4 components, ligustrazine, chlorogenic acid, quercetin and emodin were identified by comparing the retention time and UV spectra with reference standard. In addition, HPLC fingerprint was also established to assess the quality of FTP. The developed method was proved to be accurate and efficient for profiling major components in FTP.

\section{MATERIALS AND METHODS}

16 reference standards were purchased from the National Institute for the Control of Pharmaceutical and Biological Products, Beijing, China. FTP (National approval Number: Z51022427, Batch Number: 12080008, 12090010, 55110003, 55110011, 55120002, 55120003, 55120004 and 55120005) were obtained from Taiji Group, Sichuan Mianyang Pharmaceutical Co., Ltd, Mianyang, China.

HPLC grade acetonitrile, analytical grade methanol and ethanol, the main substances used for this study, came from Guangdong Guanghua Sci-Tech Co., Ltd (Shantou, China) and Kermel chemical reagents company (Tianjin, Chian), respectively.

\section{Chromatographic conditions:}

HPLC chromatographic separation was achieved by a Shimadzu model 20A liquid chromatographic system (Tokyo, Japan), equipped with a 20AT pump and a PDA detector (SPD-20A). The system was controlled by a system controller (SCL-20A) and a personal computer. A Diamonsil C18 analytical column $(250 \times 4.6 \mathrm{~mm}$, $5 \mu \mathrm{m})$ and a guard column $(10 \times 4.0 \mathrm{~mm}, 5 \mu \mathrm{m})$ were utilized. The column temperature was maintained at $30^{\circ}$. The mobile phase A was consisted of acetonitrilewater $(5: 95, \mathrm{v} / \mathrm{v}$, containing $0.08 \%$ trifluoroacetic acid) and mobile phase B was acetonitrile-water $(95: 5, \mathrm{v} / \mathrm{v}$, containing $0.08 \%$ trifluoroacetic acid). The gradient elution program was as follows: 0 to $4 \% \mathrm{~B}$ from 0 to $5 \mathrm{~min}, 4 \% \mathrm{~B}$ from $5-10 \mathrm{~min}, 4$ to $12 \% \mathrm{~B}$ from 10 to $25 \mathrm{~min}, 12$ to $13 \%$ B from 25 to $30 \mathrm{~min}, 13$ to $19 \% \mathrm{~B}$ from 30 to $40 \mathrm{~min}, 19$ to $24 \%$ B from 40 to $52 \mathrm{~min}, 24$ to $25 \%$ B from 52 to $60 \mathrm{~min}, 25$ to $42 \%$ B from 60 to $70 \mathrm{~min}, 42$ to $46 \%$ B from 70 to $90 \mathrm{~min}, 46$ to $86 \% \mathrm{~B}$ from 90 to $130 \mathrm{~min}, 86$ to $0 \% \mathrm{~B}$ from 130 to $140 \mathrm{~min}$. 
The flow rate was $1.0 \mathrm{ml} / \mathrm{min}$ and the injection volume was $20 \mu \mathrm{l}$. The detection wavelength was set at $254 \mathrm{~nm}$.

\section{Preparation of solutions:}

FTP preparation was ground into powder and then passed through a 20 mesh sieve and a 60 mesh sieve successively. $1.0 \mathrm{~g}$ middle particle size powder was extracted by $15 \mathrm{ml}$ methanol using ultrasonic bath for $40 \mathrm{~min}$ at $60^{\circ}$. The extract was centrifuged at $15000 \mathrm{rpm}$ for $5 \mathrm{~min}$, and then supernatant was filtered through $0.45 \mu \mathrm{m}$ micropore film before injecting $20 \mu \mathrm{l}$ into the HPLC system for analysis.

All standard solutions were prepared by methanol and the stock solutions were stored at $4^{\circ}$ in refrigerator. Appropriate volumes of each standard stock solution were diluted with methanol to make different concentrations. The concentrations of 16 kinds of standard solution in quantitative analysis of ligustrazine, ephedrine, chlorogenic acid, geniposide, ferulicacid, 5-O-methylvisammioside, hesperidin, baicalin, quercetin, paeonol, glycyrrhizic acid, aloeemodin, rhein, emodin, chrysophanol, physcion, were $0.00387,0.170,0.0533,0.0716,0.0810,0.0853$, $0.0213,0.208,0.167,0.015,0.2933,0.0036,0.00174$, $0.0024,0.0286$ and $0.0049 \mathrm{mg} / \mathrm{ml}$, respectively.

\section{Quantitative determination by HPLC-PDA:}

Gradient elution HPLC method was developed to profile the major components and determine twelve bioactive markers in FTP quantitatively. The method was validated by aspects of specificity, linearity and range, limit of detection (LOD) and limit of quantitation (LOQ), precision and accuracy, according to the requirements of $\mathrm{ChP} .2010$.

\section{Similarity matches of the HPLC fingerprinting chromatograms:}

Similarity evaluation system for chromatographic fingerprint of TCM (Version 2004 A, by National pharmacopoeia committee) was used to evaluate the similarity and identify common peaks of eight batches of FTP (Batch Number: 12080008, 12090010, 55110003, 55110011, 55120002, 55120003, 55120004 and 55120005).

\section{RESULTS AND DISCUSSION}

Sample extraction conditions, such as extraction method, extraction solvent type, extraction time, extraction temperature, the ratio of extraction solution volume to the amount of sample and the particle size method were designed by univariate factor experimental. The percentage yields of 16 selected markers as well as the number of chromatographic peak were used as the markers for efficiency evaluation.

Ultrasonic extraction was chosen because of its higher extraction efficiency and shorter extraction time than reflux extraction. Methanol, ethanol, hydrous methanol or ethanol (90, 70 and $50 \%$ ), ethyl acetate and water as extraction solvents were investigated. The results showed methanol as solvent could obtain higher extraction yield of the selected markers and extract more ingredients. Due to FTP containing acid and alkaline compounds, acid or alkaline was added to extraction solvent in order to obtain better resolution, peak shape and higher extract yield. However, the above parameters had no significant change. Moreover, baicalin was unstable and would degrade into baicalein under the action of ultrasound and acid.

The extraction time, extraction temperature and the ratio of extraction solvent volume to the amount of sample were also optimized. The condition of 40 min, $15 \mathrm{ml}$ extraction solvent to $1.0 \mathrm{~g}$ sample at $60^{\circ}$ was found to give a complete extraction and higher extraction yields. Temperature higher than $60^{\circ}$ was not tested considering the boiling point of methanol $\left(65.4^{\circ}\right)$.

Sample uniformity has great influence on the repeatability of determination results, and the smaller the particle size is, the more uniform the sample is. On the other hand, the extraction yields of selected markers were also affected by the particle size. The study showed that too small size of sample would lower the rate of extraction. The reason would be that high sugar content in FTP made the solution sticky and restricted the dissolution of components, when sample was ground too fine. After optimization, the powders passed through a 20 mesh sieve and a 60 mesh sieve successively was finally chosen.

The chromatogram of the samples processed by the optimized pretreatment method described in Preparation of solutions section displayed higher extraction efficiency, shorter extraction time and more extraction of the samples (Fig 1).

Gradient elution HPLC method was adopted due to the complexity of the prescription and the great differences of the components' chemical properties in FTP. For optimizing the constituents of the mobile phase, acetonitrile-water, acetonitrile-aqueous phosphoric acid, acetonitrile-aqueous trifluoroacetic acid and 


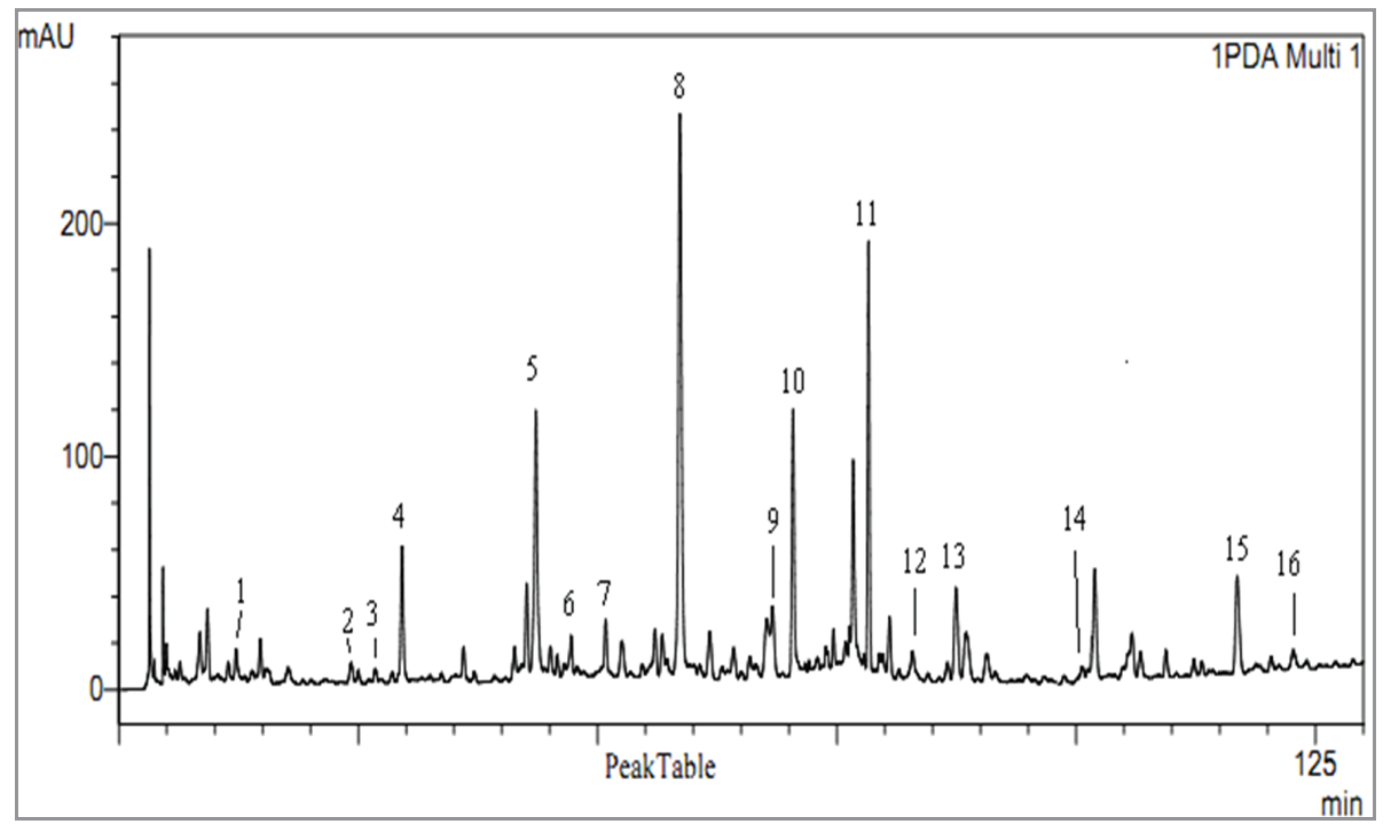

Fig. 1: Chromatogram of FTS.

1. ligustrazine, 2. ephedrine, 3. chlorogenic acid, 4. geniposide, 5. ferulic acid, 6. 5-O-methylvisammioside, 7. hesperidin, 8. baicalin, 9. quercetin, 10. paeonol, 11. glycyrrhizic acid, 12. aloe-emodin, 13. rhein, 14. emodin, 15. chrysophanol, 16. physcion

acetonitrile-aqueous acetic acid in different mobile phase systems were adopted in order to achieve the best separation. The results showed that mobile system with acetonitrile $-0.08 \%$ trifluoroacetic acid could produce a steady baseline and symmetrical peak. The gradient time and gradient slope were also adjusted. Most of the compounds were found to be separated in a single HPLC run with baseline separation under the elution program listed in Chromatographic conditions section. Five different wavelengths (210, 254, 283, 340 and $430 \mathrm{~nm}$ ) were investigated by HPLC-PDA system to display more peaks in the fingerprint. The results suggested that the components of interest showed strong UV absorbance and the number of peaks was the most when detection wavelength was set at 254 $\mathrm{nm}$. Therefore, $254 \mathrm{~nm}$ was chosen for obtaining the chromatographic fingerprint profile.

The specificity of the developed method was evaluated by comparing UV spectra and the retention time of 16 bioactive compounds with reference standards, ligustrazine, ephedrine, chlorogenic acid, geniposide, ferulic acid, 5-O-methylvisammioside, hesperidin, baicalin, quercetin, paeonol, glycyrrhizic acid, aloeemodin, rhein, emodin, chrysophanol, physcion, which were about 13.2, 25.0, 27.7, 30.2, 44.3, 47.9, 51.8, 59.0, 69.0, 76.5, 78.0, 83.1, 86.6, 104.5, 116.6 and $122.5 \mathrm{~min}$, respectively. The shape of peaks was perfect, and the peak purity indexes met the requirements (all over 0.99 ).

May - June 2016

Indian Journal of Pharmaceutical Sciences
The linearity was analyzed by 5 serial concentrations of each selected marker standard solutions at their maximum absorption wavelength, respectively. The regression equations were established by peak areas versus concentrations. LOD and LOQ were used to reflect the sensitivity of instrument and developed method. LOD was determined by a signal-to-noise ratio of 3 and LOQ was to a signal-to-noise ratio of 10 . The results were presented in Table 1.

Precision was used to evaluate the stability of instrument and the error of operation and injection. The relative standard deviation (RSD) values of injection precision for standard solutions ranged from $0.62-1.86 \%$, which were below the provided limitation of $2.0 \%$ by Ch.P.2010. Repeatability or intra-day precision was investigated by injecting 6 replicate sample solutions on the same day. Inter-day precision was assessed by analyzing newly prepared sample solutions triplicate over three consecutive days. The RSD values ranged from 0.93 to $3.37 \%$ for intra-day precision and 1.41 to $7.81 \%$ for inter-day precision, respectively. The results showed that the precision of the method was good.

The accuracy of the developed method was assessed by the method of standard additions. In brief, $0.5 \mathrm{~g}$ ground sample was spiked with a known amount of each reference compound by adding standard stock solutions. The mixture was then processed as described above $(\mathrm{n}=6)$. The recovery results showed in Table 2 implied that the method was accurate. 
TABLE 1: LINEARITY AND RANGE, LOD, LOQ

\begin{tabular}{cccccc}
\hline Analyte & Linearity range $(\mu \mathrm{g} / \mathrm{ml})$ & Regression equation & $\begin{array}{c}\text { Correlation } \\
\text { coefficient }\end{array}$ & LOD $(\mathrm{ng})$ LOQ $(\mathrm{ng})$ \\
\hline Ligustrazine & $2.32-9.28$ & $\mathrm{y}=25884 \mathrm{x}-7448$ & 0.9997 & 0.31 & 0.97 \\
Ephedrine & $102.00-680.00$ & $\mathrm{y}=838.06 \mathrm{x}-136.5$ & 0.9994 & 36.6 & 109.8 \\
Chlorogenic acid & $26.70-93.30$ & $\mathrm{y}=5975.8 \mathrm{x}-2146.7$ & 0.9996 & 0.32 & 0.99 \\
Geniposide & $44.00-110.00$ & $\mathrm{y}=20102 \mathrm{x}+1035.8$ & 0.9991 & 0.03 & 0.10 \\
Ferulic acid & $43.20-124.20$ & $\mathrm{y}=53223 \mathrm{x}+3560.4$ & 0.9997 & 1.17 & 3.51 \\
5-0-methylvisammioside & $32.00-160.00$ & $\mathrm{y}=143.24 \mathrm{x}-738.61$ & 0.9992 & 2.26 & 6.78 \\
Hesperidin & $10.67-32.00$ & $\mathrm{y}=21674 \mathrm{x}-28192$ & 0.9990 & 0.70 & 2.80 \\
Baicalin & $104.00-312.00$ & $\mathrm{y}=56607 x-4242.2$ & 0.9997 & 0.08 & 0.26 \\
Quercetin & $55.70-557.3$ & $\mathrm{y}=240.4 \mathrm{x}-121.56$ & 0.9995 & 0.51 & 1.47 \\
Paeonol & $10.75-53.74$ & $\mathrm{y}=138521 \mathrm{x}+21553$ & 0.9997 & 0.61 & 1.95 \\
Glycyrrhizic acid & $108.00-432.00$ & $\mathrm{y}=7042 \mathrm{x}+4143$ & 0.9994 & 2.20 & 6.60 \\
Aloe-emodin & $1.44-9.36$ & $\mathrm{y}=60366 \mathrm{x}+15188$ & 0.9996 & 0.19 & 0.54 \\
Rhein & $1.70-14.28$ & $\mathrm{y}=57617 \mathrm{x}+3296.3$ & 0.9999 & 0.17 & 0.51 \\
Emodin & $0.80-12.00$ & $\mathrm{y}=1792.2 \mathrm{x}+118.8$ & 0.9994 & 0.19 & 0.49 \\
Chrysophanol & $5.64-28.20$ & $\mathrm{y}=58191 \mathrm{x}+8149.4$ & 0.9994 & 0.45 & 1.41 \\
Physcion & $0.98-5.88$ & $\mathrm{y}=52330 \mathrm{x}+1691.1$ & 0.9993 & 0.02 & 0.05 \\
\hline
\end{tabular}

TABLE 2: RECOVERY RESULT OF SIXTEEN MARKERS

\begin{tabular}{lccccc}
\hline Analyte & Original $(\mu \mathrm{g})$ & Spiked $(\mu \mathrm{g})$ & Found $(\mu \mathrm{g})$ & Recovery $(\%)$ & RSD $(\%)$ \\
\hline Ligustrazine & 0.04 & 0.04 & 0.077 & 92.50 & 4.95 \\
Ephedrine & 1.66 & 1.28 & 2.86 & 95.20 & 2.52 \\
Chlorogenic acid & 0.34 & 0.40 & 0.73 & 97.00 & 6.76 \\
Geniposide & 0.62 & 0.66 & 1.30 & 103.20 & 1.15 \\
Ferulic acid & 0.50 & 0.61 & 1.10 & 98.00 & 1.73 \\
5-O-methylvisammioside & 0.62 & 0.64 & 1.30 & 106.40 & 3.65 \\
Hesperidin & 0.14 & 0.16 & 0.31 & 107.10 & 1.04 \\
Baicalin & 1.66 & 1.56 & 3.20 & 98.80 & 1.04 \\
Quercetin & 1.10 & 1.25 & 2.26 & 91.80 & 4.03 \\
Paeonol & 0.15 & 0.16 & 0.32 & 106.70 & 1.29 \\
Glycyrrhizic acid & 2.12 & 2.16 & 4.32 & 101.90 & 1.85 \\
Aloe-emodin & 0.03 & 0.03 & 0.062 & 106.70 & 2.68 \\
Rhein & 0.014 & 0.012 & 0.027 & 107.10 & 3.78 \\
Emodin & 0.02 & 0.02 & 0.039 & 95.00 & 3.44 \\
Chrysophanol & 0.20 & 0.21 & 0.42 & 105.00 & 1.50 \\
Physcion & 0.04 & 0.04 & 0.076 & 90.00 & 3.20 \\
\hline
\end{tabular}

$\mathrm{N}=6$, Batch Number: 12080008. ${ }^{\text {a. }}$ the amount of Glycyrrhizic acid would multiply by 0.98 , which was the ratio of the molecular weight between Glycyrrhizic acid and Monoammonium glycyrrhizate.

The stability of sample solutions was assessed by the RSD of peak areas of each markers standing for $48 \mathrm{~h}$. The RSD values ranged from 0.16 to $1.89 \%$, indicating that the sample solution was stable within $48 \mathrm{~h}$. Despite this, quantitative analysis was conducted within $24 \mathrm{~h}$ after sample preparation in order to avoid any possible decomposition.

The method was validated with respect to variation of the instrument characteristics. Two different brand HPLC instruments (Shimadzu model 20A and Ultimate $3000)$ were used. There were no significant changes in chromatographic window. The chromtographic profile was similar and the selected markers were all displayed in chromatogram. The results showed that the method was robust.

8 batches of FTP were analyzed using the developed method. The result showed that these products possess similar chromatographic profiles (fig 2). The similarity evaluation system for chromatographic fingerprint of TCM (Version 2004 A, by National Pharmacopoeia Committee) was used in the similarity study of chromatographic and spectral profiles. The 


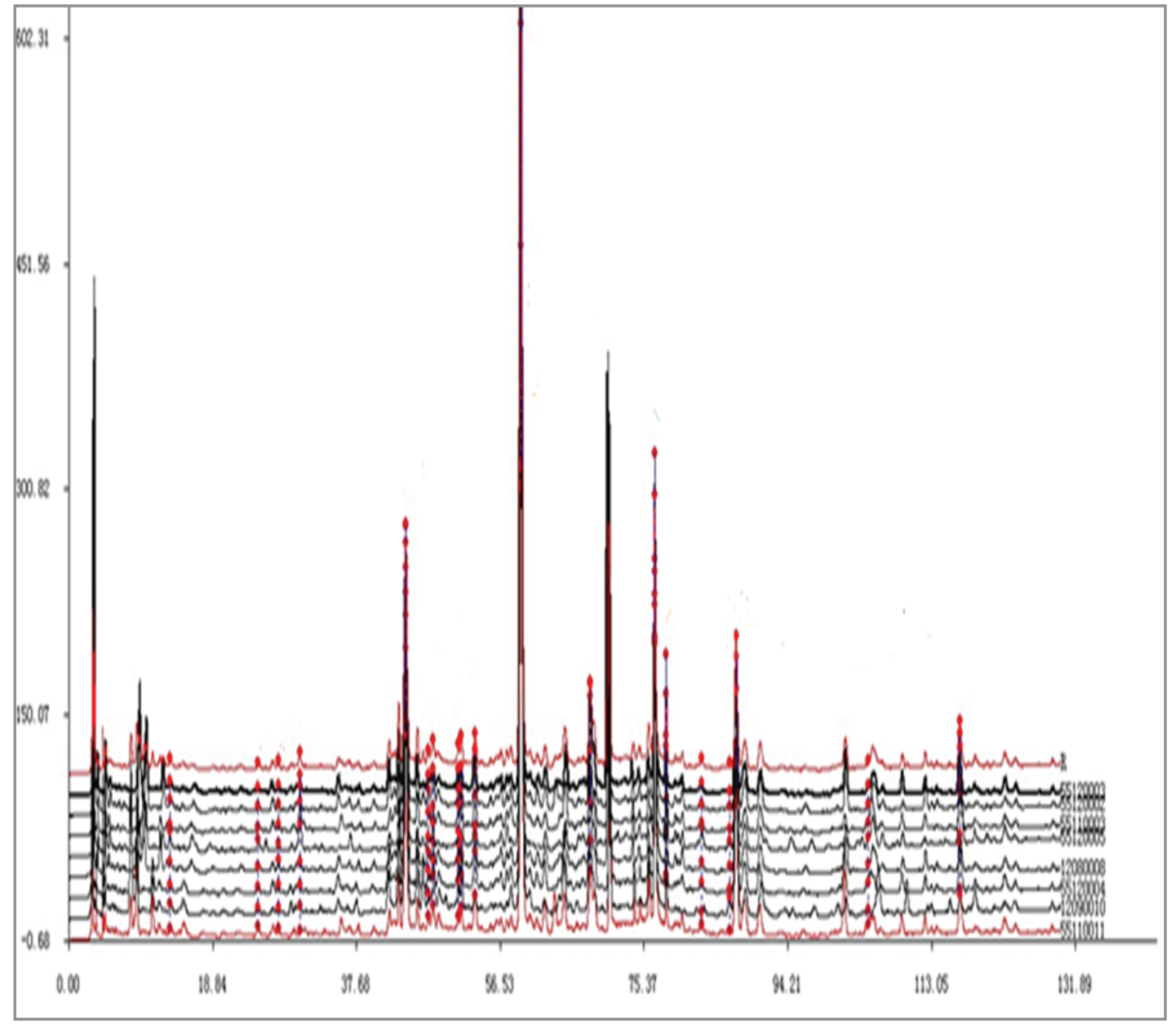

Fig. 2: The fingerprint of eight batches of FTS.

simulative mean chromatogram of FTP was generated and the correlation coefficients of chromatograms were calculated. The similarity match of chromatograms showed that the profiles of 8 batches of FTP were consistent to each other although the peak intensities were somewhat different. This might be a result of the variation in the quality of raw materials. The correlation coefficient of eight sample chromatograms to their simulative mean chromatogram ranged from 0.934 to 0.999 , indicating that the quality of different bathes of FTP from the same manufacturer was stable. To further evaluate the quality, the contents of the selected markers were studied. Although sixteen components were identified by comparing the retention time and UV spectra with reference standard, only twelve were assayed and the other six components wasn't assayed because their contents were too low or were not well separated. The results of quantitative determination were listed in Table 3.

At present, the quality control of TCM patent prescriptions, the critical challenges lie in the complex chemical matrices and the variety of chemical structures. The HPLC fingerprint profiles we applied be used to comprehensively analyze the type information of TCM and content distribution of the effective and ineffective components in traditional Chinese herbal preparation, in accordance with the characteristics of the integral and fuzzy characteristics of Chinese Medicine ${ }^{[19]}$. The established method determining a complex traditional Chinese herbal preparation, FTP, by HPLC with photodiode array detection, succeeded in quantitative determination of 12 active components, and qualitative identification 4 ingredients, simultaneously. The developed method was validated according to Ch.P.2010. The results showed the method was accurate and precision and the manufacturing process and quality of FTP was stable. This 'multicomponents' quantification and fingerprint profile method could be used to evaluate the quality of FTP and could be done together.

As more and more technologies have been applied to the fingerprint research of TCM, An inevitable trend that it played a much more important role in many aspects, such as the quality control, the researches of 
TABLE 3: RESULTS OF QUANTITATIVE DETERMINATION BY HPLC

\begin{tabular}{ccccccccc}
\hline Analyte & \multicolumn{7}{c}{ Found $(\mathrm{mg} / \mathrm{g})$} \\
\cline { 2 - 9 } & B1 & B2 & B3 & B4 & B5 & B6 & B7 & B8 \\
\hline Ligustrazine & 0.07 & 0.13 & 0.06 & 0.14 & 0.08 & 0.07 & 0.14 & 0.10 \\
Ephedrine & 3.12 & 2.19 & 2.69 & 4.33 & 2.91 & 2.94 & 3.62 & 3.43 \\
Chlorogenic Acid & 0.65 & 0.50 & 0.38 & 0.47 & 0.53 & 0.63 & 0.67 & 0.70 \\
Geniposide & 1.16 & 0.91 & 0.84 & 0.83 & 1.25 & 1.31 & 1.39 & 1.24 \\
Ferulic Acid & 0.92 & 1.63 & 0.82 & 1.10 & 1.32 & 1.24 & 1.51 & 1.00 \\
5-O-methylvisammioside & 1.16 & 1.16 & 1.18 & 1.05 & 1.49 & 0.95 & 0.82 & 1.18 \\
Hesperidin & 0.26 & 0.15 & 0.34 & 0.39 & 0.41 & 0.42 & 0.38 & 0.29 \\
Baicalin & 3.13 & 3.80 & 3.06 & 2.57 & 3.64 & 3.39 & 3.96 & 3.36 \\
Quercetin & 2.08 & 1.32 & 2.05 & 2.42 & 2.46 & 2.56 & 2.36 & 2.02 \\
Paeonol & 0.28 & 0.38 & 0.28 & 0.38 & 0.39 & 0.23 & 0.28 & 0.34 \\
Glycyrrhizic acid & 3.98 & 2.58 & 3.48 & 3.49 & 4.33 & 3.78 & 4.08 & 4.19 \\
Aloe-emodin & 0.05 & 0.05 & 0.05 & 0.08 & 0.04 & 0.06 & 0.09 & 0.07 \\
Rhein & 0.03 & 0.10 & 0.02 & 0.03 & 0.04 & 0.03 & 0.03 & 0.03 \\
Emodin & 0.03 & 0.04 & 0.03 & 0.03 & 0.02 & 0.04 & 0.03 & 0.03 \\
Chrysophanol & 0.36 & 0.12 & 0.31 & 0.35 & 0.20 & 0.36 & 0.39 & 0.39 \\
Physcion & 0.07 & $\mathrm{~N}^{\mathrm{a}}$ & 0.05 & 0.07 & 0.04 & 0.07 & 0.08 & 0.07 \\
\hline
\end{tabular}

B1 to B8 refer to eight different batches. arefers to not detected.

efficient components, and the mechanism in TCM, and so on ${ }^{[20]}$.

\section{Acknowledgements:}

We would like to show appreciation to our teachers from College of Pharmacy for providing technical support and experimental equipment.

\section{Financial support and sponsorship:}

This research was supported by Sichuan Province Natural Science Foundation (No.2014JY01118).

\section{Conflicts of interest:}

There are no conflicts of interest.

\section{REFERENCES}

1. Mok DKW, Chau FT. Chemical information of Chinese medicines: A challenge to chemist. Chemometr Intell Lab Sys 2006;82:210-7.

2. Jiang Y, David B, Tu PF, Barbin Y. Recent analytical approaches in quality control of traditional Chinese medicines-A review. Anal Chim Acta 2010;657:9-18.

3. Shang ZC. Fangfeng Tongsheng pills. Family Medicine 1998;18:22-3.

1. Yoshida T, Sakane N, Wakabayashi Y, Umekawa T, Kondo M. Thermogenic, antiobesity effects of bofu-tsusho-san in MSGobese mice. Int J Obes Relat Metab Disord 1995;19:717-22.

2. Akiko O, Michiko S, Yasuo M, Shigeru T. Effects of Bofutsusho-san (Fang-Feng-Tong-Sheng-San) on body weight and fat content in normal mice. J Trad med 2001;18:33-8.

3. Morimoto Y, Sakata M, Ohno A, Maeqawa T, Tajima S.
Effects of bofu-tsusho-san, a traditional Chinese medicine, on body fat accumulation in fructose-loaded rats. Yakurigaku Zasshi 2001;117:77-86.

4. Morimoto Y, Sakata M, Ohno A, Maegawa T, Tajima S. Effects of Byakko-ka-ninjin-to, Bofu-tsusho-san and Goreisan on blood glucose level, water intake and urine volume in KKAy mice. Yakugaku Zasshi 2002;122:163-8.

5. Yi SS, Yao LW. Determination of Baicalin in Fangfeng Tongsheng Pill by HPLC. China Pharm 2007;21:902-3.

6. Zhao PF, Cai XS, Zhao XB. Determination of forsythin in Fangfeng tongsheng Pill by HPLC. J Chin Med 2009;24:1456.

7. Liu AJ, Qi GC, Liu ZY, Bai ZJ. Simultaneous determination of paeoniflorin, geniposide, baicalin and forsythin in Fangfeng tongsheng Pills by RP-HPLC. Northwest Pharm J 2012;27:415-7.

8. Li W, Wang Z, Chen L, Zhang J, Han L, Hou J, et al. Pressurized liquid extraction followed by LC-ESI/MS for analysis of four chromones in Radix Saposhnikoviae. J Sep Sci 2010;33:2881-7.

9. Powell T, Hsu FF, Turk J, Hruska K. Ma-huang strikes again: Ephedrine nephrolithiasis. Am J Kidney Dis 1998;32:153-9.

10. Feng L, Xiong Y, Cheng F, Zhang L, Li S, Li Y. Effect of ligustrazine on ischemia-reperfusion injury in murine kidney. Transplant Proc 2004;36:1949-51.

11. Suzuki Y, Kondo K, Ikeda Y, Umemura K. Antithrombotic effect of geniposide and genipin in the mouse thrombosis model. Planta Med 2001;67:807-10.

12. Deng CH, Yao N, Wang B, Zhang XM. Development of microwave-assisted extraction followed by headspace singledrop microextraction for fast determination of paeonol in traditional Chinese medicines. J Chromatogr A 2006;1103:15-21.

13. Christopher RH, Perry AM, Praveen KS. Identification and quantification of eight flavones in root and shoot tissues of the medicinal plant Huang-qin (Scutellaria baicalensis Georgi) 
www.ijpsonline.com

using high-performance liquid chromatography with diode array and mass spectrometric detection. J Chromatogr A 2005;1062:199-207.

14. Cai YZ, Luo Q, Sun M, Corke H. Antioxidant activity and phenolic compounds of 112 traditional Chinese medicinal plants associated with anticancer. Life Sci 2004;74:2157-84.

15. Li Y, Liu HW, Ji XH, Li JL. Optimized separation of pharmacologically active anthraquinones in Rhubarb by capillary electrochromatography. Electrophoresis 2000;21:3109-15.

16. Sun L, Qiao SY, Zhao YM. Fingerprint of traditional Chinese materia medica: advances in applied researches. J Int Pharm Res 2009; 3:194-203.

17. Li Q, Du SM, Zhang ZL, LV CM, Zhou YQ, Zhao Y, Zhang N. Progress in fingerprint technology on Chinese materia medica and prospect of its future development. Chin Tradit Herb Drugs 2013;44:3095-104. 